DOI: https://doi.org/10.46296/yc.v5i9edespsoct.0115

\title{
PLATAFORMAS TECNOLÓGICAS Y SU APORTE AL APRENDIZAJE EN LÍNEA PARA LA ASIGNATURA DE MATEMÁTICA
}

\section{TECHNOLOGICAL PLATFORMS AND THEIR CONTRIBUTION TO ONLINE LEARNING FOR THE MATHEMATICS SUBJECT}

\author{
Morán-González Miguel ${ }^{1}$; Gallegos-Macías Marcos Ramón ${ }^{2}$ \\ ${ }^{1}$ Universidad San Gregorio de Portoviejo USGP. Portoviejo Ecuador. Correo: \\ mimogo_28@hotmail.com. \\ 2 Universidad San Gregorio de Portoviejo USGP. Portoviejo Ecuador. Correo: \\ mgallegos@sangregorio.edu.ec. ORCID ID: https://orcid.org/0000-0002-3651-034x
}

\begin{abstract}
Resumen
El presente estudio abarca el análisis de las plataformas tecnológicas utilizadas durante el proceso enseñanza y aprendizaje de matemática. El objetivo es identificar el dominio en el uso de herramientas y plataformas tecnológicas en la educación en línea en la asignatura de matemática específicamente en el primero de bachillerato de la unidad educativa San Mateo. La metodología fue de enfoque cuanti-cualitativo, es decir, que se abordó el tema de estudio desde los aspectos cuantitativos y medibles, con la finalidad de identificar hallazgos de importancia que sirvan de base a la investigación en lo relativo a la aplicación de las plataformas tecnológicas en el área de matemática. Las herramientas que se utilizaron fueron entrevista dirigida a expertos y encuesta a docente y estudiantes. Se valoró plataformas tecnológicas que favorecen la innovación en los procesos de enseñanza y de aprendizaje, en modalidad virtual (Silva Quiroz, 2010). Los resultados indican que los docentes carecen del dominio de herramientas y plataformas tecnológicas y se evidencia que los estudiantes reciben sus clases solo a través de video conferencias, descuidando las actividades interactivas que ofrecen las plataformas tecnológicas, sin embargo, tanto docente como estudiante muestran interés en aprender, esto debido a la necesidad que implica la enseñanza a través de la internet, se concluye además exponiendo algunos criterios a tomar en cuenta para potenciar el uso de estas herramientas en la institución educativa objeto de estudio.
\end{abstract}

Palabras claves: Plataformas tecnológicas, aprendizaje de matemáticas; aprendizaje en línea, herramientas informáticas.

\begin{abstract}
This study covers the analysis of the technological platforms used during the teaching and learning process of mathematics. The objective is to identify the domain in the use of technological tools and platforms in online education in the subject of mathematics specifically in the first year of high school at the San Mateo educational unit. The methodology was quantitative-qualitative approach, that is, the subject of study was approached from the quantitative and measurable aspects, in order to identify important findings that serve as a basis for the research regarding the application of the platforms. technological in the area of mathematics. The tools used were an interview directed at experts and a survey of teachers and students. Technological platforms that favor innovation in teaching and learning processes, in virtual mode, were valued (Silva Quiroz, 2010). The results indicate that teachers lack the mastery of technological tools and platforms and it is evidenced that students receive their classes only through video conferences, neglecting the interactive activities offered by technological platforms, however, both teacher and student show interest in learn, this due to the need that teaching through the internet implies, it is also concluded by exposing some criteria to take into account to enhance the use of these tools in the educational institution under study.
\end{abstract}

Keywords: Technology platforms, math learning; online learning, computer tools.

Información del manuscrito:

Fecha de recepción: 22 de julio de 2021.

Fecha de aceptación: 24 de septiembre de 2021.

Fecha de publicación: 01 de octubre de 2021. 


\section{Introducción}

Las tecnologías de la información y la comunicación

(TIC's)

conjuntamente con la educación contribuyen a la existencia de un acceso universal a la formación profesional por lo cual son de vital importancia en el proceso de enseñanza sin embargo el deficiente manejo del área de matemáticas en las plataformas tecnológicas educativas conlleva a la existencia de un problema en el aprendizaje de esta área por aquello es muy importante identificar las diferentes herramientas y plataformas tecnológicas que permitan una correcta enseñanza de las matemáticas. La presente investigación se fundamenta en analizar las plataformas tecnológicas y herramientas que faciliten el aprendizaje de las matemáticas para el docente y el estudiante logrando que puedan desarrollar conocimientos en un ambiente motivador favoreciendo en el proceso de enseñanza y de aprendizaje.

Cobos \& Torres (2017):

La educación tiene como vehículo para lograr sus fines el uso sistemático de la tecnología educativa, que establece la manera de planificarla y ponerla en práctica: al configurar los procesos de enseñanza $y$ aprendizaje, sus recursos, espacios y tiempos. El discurso pedagógico superpone el concepto de tecnología educativa (tecnologías de la educación), al de tecnologías de la información y la comunicación (tecnologías en la educación). (Pág. 38)

El autor explica el inconveniente que tiene el docente al momento de explicar su clase en línea tras la problemática que surge al no saber manejar las herramientas y plataformas de una forma que le permitan un desarrollo adecuado. Conjuntamente, recalca la problemática existente de parte del alumnado debido a la existencia de familias de condición económica baja lo cual impide a los estudiantes acceder a ordenadores o teléfonos de alta tecnología siendo recursos necesarios para las clases en línea.

\section{Las Plataformas Tecnológicas (PT) en la educación.}

Según Campos \& Boulet (2016): 
Las plataformas tecnológicas tienen en la contemporaneidad un amplio uso en el sector empresarial, comercial $y$ educativo. En el sector educativo las PT integran una amplia variedad de aplicaciones informáticas para facilitar la formación de los estudiantes y su acceso a un conjunto de recursos y herramientas útiles en cada momento y localidad específica. En el ámbito universitario las PT propician la gestión de la calidad de los procesos y funciones sustantivas propias de este nivel. (Pág. 6)

El mundo ha sido afectado por el aislamiento dado como medida de prevención tras la pandemia del covid 19. La educación fue una de las áreas más afectadas, debido a que fue necesario suspender las actividades presenciales en centros de educación inicial, medio y superior, forzando a realizar el proceso de enseñanza aprendizaje a través de la tecnología, tanto estudiantes como profesores tuvieron que adaptarse a estos nuevos procesos de formación educativa.
Las plataformas educativas permiten al docente crear un espacio virtual, donde se configuran herramientas y recursos digitales necesarios para desarrollar el proceso de enseñanza y de aprendizaje, más aún en la virtualidad o educación en línea.

Para Fernández \& Rivero (2014):

Los entornos virtuales a través de plataformas de aprendizajes, están presentes en la actualidad de forma cotidiana en los procesos de enseñanza $y$ de aprendizaje, con el objetivo de elevar la calidad del proceso educativo y hacerlo más accesible, eliminando las barreras de espacio y tiempo, siendo innumerables las universidades a nivel internacional que disponen de campus virtuales y enseñanza en línea. (Pág. 208)

Las plataformas virtuales se han convertido en eje fundamental para la impartición de una clase, por ser el canal del proceso de enseñanza para el docente en el desarrollo de su materia. Se entiende por plataforma educativa, un sitio web que permite al docente contar con un espacio virtual, que contenga recursos y materiales de apoyo que 
le permitan desarrollar su conferencia académica y que acceda a foros, wikis, recibir tareas del estudiante, desarrollar test, promover debates, chats y obtener estadísticas de evaluación, entre otros recursos que crea necesarios para poder incluir en su curso, a partir de un diseño previo, que reconozca actividades de aprendizaje y que ayude a sus estudiantes a lograr los objetivos planteados en el currículo del curso .
Existen diferentes plataformas educativas en la web, en las que el docente puede desarrollar sus clases, pero existe una barrera cuando se asigna una plataforma al docente y no tiene la opción de escoger la mejor plataforma educativa para dictar su materia.

\section{Plataformas de Software Libre}

Según Sánchez (2005) las plataformas más utilizadas y plantea información general que se expone en el siguiente cuadro:

Cuadro 1. Plataformas tecnológicas para enseñanza.

\begin{tabular}{|c|l|}
\hline PLATAFORMAS & \multicolumn{1}{c|}{ GENERALIDADES } \\
\hline Moodle & $\begin{array}{l}\text { Actualmente existen 12.892 sitios Web que la utilizan en 158 países. } \\
\text { Está traducida a más de 70 idiomas. Teniendo en cuenta que Moodle } \\
\text { fue iniciada en 1999 y que la primera versión salió en 2002, las cifras } \\
\text { anteriores nos dan idea del crecimiento exponencial de esta } \\
\text { plataforma. Va camino de convertirse en un estándar de plataforma } \\
\text { educativa virtual, con usuarios tan prestigiosos como la británica Open } \\
\text { University. }\end{array}$ \\
\hline Dokeos & $\begin{array}{l}\text { Está siendo utilizada por más hv. de 1.000 organizaciones } \\
\text { (universidades, institutos, administraciones públicas y empresas) en } \\
\text { más de 63 países y 34 idiomas. }\end{array}$ \\
\hline Claroline & $\begin{array}{l}\text { Administraciones y organismos se está haciendo en este sentido, pues } \\
\text { se permite hacer una recopilación del mismo y distribuirla entre el } \\
\text { alumnado y el profesorado, para que en casa dispongan y trabajen con } \\
\text { las mismas aplicaciones que en su centro educativo (hecho que sería } \\
\text { inviable con el software propietario). }\end{array}$ \\
\hline
\end{tabular}

Moodle es una plataforma virtual con un diseño eficiente y seguro que brinda diferentes beneficios para sus usuarios, el hecho de ser una herramienta gratuita y multilingüe ya es una gran ventaja, por otro lado merece destacar su facilidad de uso, compatibilidad con dispositivos móviles y con una gran variedad de herramientas. Moodle ha demostrado ser eficiente en su implementación en el aprendizaje y 
la enseñanza, además de adaptarse

a las necesidades del usuario.

\section{Beneficio de plataformas tecnológicas en la educación}

Según Cañar, Andrango \& Muso (2021):

Las plataformas virtuales, constituye un recurso formativo que contribuye a la mejora de la enseñanza aportando herramientas telemáticas

facilitadoras y promotoras de un aprendizaje constructivo donde predomina la colaboración, motivación, interactividad, participación, flexibilidad, el análisis crítico-reflexivo de los contenidos $y$ materiales disponibles tanto en el campus virtual en particular, como en Internet en general. (Pág.1223)

\section{Características de las plataformas tecnológicas}

Según la Universidad Internacional de Valencia (2015) los componentes o características básicas de todo entorno virtual de aprendizaje, que además deben estar fuertemente ligados e interconectados, de forma que se influyan mutuamente y se retroalimenten pueden sintetizarse en los siguientes:

Cuadro 2. Características de las plataformas tecnológica

\begin{tabular}{|c|l|}
\hline Características & \multicolumn{1}{|c|}{ Definición } \\
\hline Estandarización & $\begin{array}{l}\text { Esta característica permite utilizar cursos realizados por terceros, } \\
\text { personalizando el contenido y reutilizando el conocimiento. }\end{array}$ \\
\hline Funcionalidad. & $\begin{array}{l}\text { Prestaciones y características que hacen que cada plataforma } \\
\text { sea adecuada (funcional) según los requerimientos y } \\
\text { necesidades de los usuarios. }\end{array}$ \\
\hline Ubicuidad & $\begin{array}{l}\text { Capacidad de una plataforma para generar tranquilidad al } \\
\text { usuario y provocarle la certeza de que todo lo que necesita lo va } \\
\text { a encontrar en dicho entorno virtual. }\end{array}$ \\
\hline Interactividad & $\begin{array}{l}\text { La persona se convierte en el protagonista de su propio } \\
\text { aprendizaje a través del autoservicio y los servicios auto guiados. }\end{array}$ \\
\hline Escalabilidad & $\begin{array}{l}\text { Estos recursos pueden funcionar con una cantidad variable de } \\
\text { usuarios según las necesidades de la organización. }\end{array}$ \\
\hline Usabilidad & $\begin{array}{l}\text { Facilidad con que las personas pueden utilizar la plataforma con } \\
\text { el fin de alcanzar un objetivo concreto. }\end{array}$ \\
\hline Integración & $\begin{array}{l}\text { Flexibilidad la plataforma puede ser adaptada tanto a los planes } \\
\text { de estudio de la institución, como a los contenidos y estilo } \\
\text { pedagógico de la organización. También permite organizar } \\
\text { cursos con gran facilidad y rapidez. }\end{array}$ \\
\hline $\begin{array}{l}\text { Las plataformas LMS deben poder integrarse con otras } \\
\text { aplicaciones empresariales utilizadas por recursos humanos y } \\
\text { contabilidad, lo que permite medir el impacto, eficacia, y sobre } \\
\text { todo, el coste de las actividades de formación. }\end{array}$ \\
\hline
\end{tabular}




\section{Experiencia exitosa del uso de plataformas tecnológicas por parte de docentes de matemáticas.}

Avalos, Vásquez \& Pereyra (2020) en base a su investigación nos da a conocer:

El estudio de naturaleza aplicada, tuvo como objetivo demostrar en qué medida, aplicar la plataforma Schoology influyó en los estudiantes del ciclo VI de secundaria en su aprendizaje correspondiente al área de matemática, haciendo uso de estrategias dinámicas y creativas, que les permitan desarrollar competencias matemáticas, como respuesta a la problemática estudiantil. La metodología utilizada responde a un enfoque cuantitativo, con un método hipotético deductivo, asimismo, se utilizó un diseño cuasi experimental, conformado por dos grupos llamados; pre test y post test; para lo cual se tomó una muestra no probabilística e intencional que comprendió a estudiantes de secundaria en un numero de 60, aplicándoles un cuestionario, de acuerdo a la prueba de normalidad. Se determinó la utilización de una prueba para muestras independientes, conocida como la $T$ de Student para contrastar las hipótesis, antes y después de la utilización de estrategias dinámicas y creativas en la aplicación de la plataforma; llegando a la siguiente conclusión: El aplicar la plataforma Schoology influyó en forma significativa para el aprendizaje en las matemáticas en los estudiantes del VI ciclo de Secundaria, San Miguel, 2019, $(T=4,737: g l=58 ; p<0,05)$. (Pág. 135)

El autor da a conocer a través de los resultados de su estudio que la plataforma Schology aplicada para las clases de Matemáticas demostró su influencia en el aprendizaje de los estudiantes y en el desarrollo de una fácil comprensión para los temas relacionados con competencia de matemática, utilizando metodologías y diseños lo que permitiendo el desarrollo cognitivo del alumno. 
Plataformas

utilizadas para el aprendizaje enseñanza de matemática.
Aulaplaneta (2015) en su artículo "25

herramientas para enseñar

Matemáticas con las TIC" da a

conocer las siguientes plataformas

tecnológicas:

Cuadro 3. 25 herramientas para enseñar Matemáticas con las TIC. Aulaplaneta (2015).

\begin{tabular}{|c|c|c|}
\hline Asignaturas & Herramientas & Definición \\
\hline \multirow{4}{*}{ Aritmética } & $\begin{array}{l}\text { Math Cilenia (en } \\
\text { inglés) }\end{array}$ & $\begin{array}{l}\text { Mini juegos para practicar las operaciones básicas, } \\
\text { destinada a alumnos de Primaria }\end{array}$ \\
\hline & $\begin{array}{l}\text { Math Jump para } \\
\text { Android e iOS }\end{array}$ & $\begin{array}{l}\text { Aplicación recomendada para Primaria que funciona } \\
\text { como un videojuego en el que el usuario maneja a un } \\
\text { robot y tiene que afrontar retos aritméticos para ir } \\
\text { avanzando niveles. }\end{array}$ \\
\hline & $\begin{array}{l}\text { Calculadoras } \\
\text { matemáticas }\end{array}$ & $\begin{array}{l}\text { Selección de diferentes tipos de calculadoras online } \\
\text { para hacer operaciones de forma rápida y sencilla. }\end{array}$ \\
\hline & Ábaco online & $\begin{array}{l}\text { Para representar diferentes números, aprender a } \\
\text { sumar de manera gráfica y trabajar las cifras de otra } \\
\text { forma. }\end{array}$ \\
\hline \multirow[t]{4}{*}{ Geometría } & Descartes & $\begin{array}{l}\text { Herramienta para crear objetos interactivos, diseñada } \\
\text { especialmente para las Matemáticas, aunque } \\
\text { aplicable también a otros temas y asignaturas. En el } \\
\text { portal del proyecto hay ejemplos y recursos creados } \\
\text { con Descartes. Además de trabajar geometría, } \\
\text { puedes crear gráficos de álgebra, estadística o } \\
\text { funciones. }\end{array}$ \\
\hline & Geogebra & $\begin{array}{l}\text { Software matemático multiplataforma para crear } \\
\text { simulaciones que relacionan el álgebra con la } \\
\text { geometría, para ayudar a los alumnos a comprender } \\
\text { los conceptos de forma visual e interactiva. Cuenta } \\
\text { con una amplia galería de recursos creados con este } \\
\text { programa. }\end{array}$ \\
\hline & $\begin{array}{l}\text { Geometría } \\
\text { Dinámica }\end{array}$ & $\begin{array}{l}\text { Página web con multitud de recursos para trabajar la } \\
\text { geometría de forma interactiva. Ofrece además } \\
\text { propuestas para funciones y gráficas, probabilidad y } \\
\text { estadística y aritmética y álgebra. }\end{array}$ \\
\hline & Dièdrom & $\begin{array}{l}\text { Aplicación didáctica que dispone de un espacio en 3D } \\
\text { donde pueden construirse piezas con volumen, } \\
\text { utilizando módulos y herramientas de dibujo. }\end{array}$ \\
\hline Álgebra & Math Papa & $\begin{array}{l}\text { Calculadora de álgebra que resuelve la ecuación paso } \\
\text { a paso, para que el alumno comprenda el proceso. } \\
\text { También incluye lecciones para aprender o repasar y } \\
\text { actividades interactivas para practicar no solo álgebra } \\
\text { sino también otros temas. }\end{array}$ \\
\hline
\end{tabular}




\begin{tabular}{|c|c|c|}
\hline & Wiris & $\begin{array}{l}\text { Aplicación online que permite construir y resolver todo } \\
\text { tipo de expresiones algebraicas. Hay una opción más } \\
\text { sencilla para Primaria. }\end{array}$ \\
\hline \multirow[t]{2}{*}{$\begin{array}{l}\text { Funciones y } \\
\text { gráficas }\end{array}$} & Desmos, & $\begin{array}{l}\text { Aplicación online para representar y estudiar } \\
\text { funciones de forma gráfica. Este video explica cómo } \\
\text { funciona y las posibilidades que ofrece. Esta guía de } \\
\text { usuario también es muy útil. Cuenta con una base de } \\
\text { datos de actividades ya creadas por profesores que } \\
\text { puedes utilizar. }\end{array}$ \\
\hline & $\begin{array}{l}\text { Algeo Graphing } \\
\text { Calculator }\end{array}$ & $\begin{array}{l}\text { Aplicación para Android con la que se pueden } \\
\text { introducir y dibujar funciones de forma sencilla desde } \\
\text { el móvil o la tableta. }\end{array}$ \\
\hline \multirow{4}{*}{ Videos } & Math TV+ & $\begin{array}{l}\text { Videos a modo de lecciones explicativas sobre } \\
\text { diversos temas de la asignatura, disponibles en inglés } \\
\text { y, en muchos casos, también en español. }\end{array}$ \\
\hline & Khan Academy & $\begin{array}{l}\text { Lecciones de Matemáticas organizadas por niveles } \\
\text { educativos y temas, para ir aprendiendo poco a poco, } \\
\text { desde lo más básico hasta lo más completo. }\end{array}$ \\
\hline & Unicoos & $\begin{array}{l}\text { Otra opción, sencilla y ordenada, de video lecciones } \\
\text { que además en algunos casos incluyen materiales } \\
\text { complementarios. }\end{array}$ \\
\hline & $\begin{array}{l}\text { Más por menos } \\
\text { y Universo } \\
\text { matemático }\end{array}$ & $\begin{array}{l}\text { Dos series incluidas dentro de La aventura del saber } \\
\text { de RTVE que incluyen documentales sobre } \\
\text { conceptos, curiosidades o personajes relacionados } \\
\text { con las Matemáticas. Algunos de los videos se } \\
\text { complementan con propuestas didácticas para } \\
\text { trabajar el tema en el aula. }\end{array}$ \\
\hline \multirow{4}{*}{$\begin{array}{l}\text { Juegos y } \\
\text { actividades } \\
\text { interactivas }\end{array}$} & $\begin{array}{l}\text { Buzzmath (en } \\
\text { inglés) }\end{array}$ & $\begin{array}{l}\text { Plataforma online creada por un equipo de profesores } \\
\text { de Matemáticas que cuenta con más de } 3.000 \\
\text { problemas matemáticos y facilita la enseñanza y el } \\
\text { aprendizaje de las matemáticas a través de ejercicios } \\
\text { interactivos y visuales. }\end{array}$ \\
\hline & $\begin{array}{l}\text { Math Game } \\
\text { Time }\end{array}$ & $\begin{array}{l}\text { Repositorio de juegos de Matemáticas de todo tipo, } \\
\text { organizados por niveles o por temas. }\end{array}$ \\
\hline & Retomates & $\begin{array}{l}\text { Plataforma de actividades para practicar Matemáticas } \\
\text { de forma divertida, a través de juegos, ejercicios y } \\
\text { exámenes que puedes personalizar. Si te registras } \\
\text { puedes guardar tus avances, crear grupos y gestionar } \\
\text { tareas. Sin registro se puede acceder igualmente a } \\
\text { todas las secciones y actividades. En el enlace ¿Qué } \\
\text { es Retomates? hay información sobre el } \\
\text { funcionamiento y las opciones de la web. }\end{array}$ \\
\hline & $\begin{array}{l}\text { Materiales } \\
\text { didácticos del } \\
\text { Proyecto Gauss } \\
\text { para secundaria } \\
\text { y primaria }\end{array}$ & $\begin{array}{l}\text { Recopilación de propuestas interactivas en Java para } \\
\text { practicar todo tipo de conceptos matemáticos. Toda la } \\
\text { web del Proyecto Gauss resulta muy útil para } \\
\text { encontrar recursos, materiales y otras ideas para el } \\
\text { aprendizaje de las Matemáticas. }\end{array}$ \\
\hline
\end{tabular}




\begin{tabular}{|c|c|c|}
\hline & Amo las mates & $\begin{array}{l}\text { Amo las mates. Completa página web con recursos, } \\
\text { juegos y material interactivo para trabajar las } \\
\text { Matemáticas en Primaria y Secundaria, organizados } \\
\text { por niveles y temas. }\end{array}$ \\
\hline \multirow[t]{4}{*}{$\begin{array}{l}\text { Matemática } \\
\text { práctica }\end{array}$} & $\begin{array}{l}\text { Sector } \\
\text { Matemática }\end{array}$ & $\begin{array}{l}\text { Sector Matemática. Sitio web con multitud de ideas } \\
\text { para aplicar las Matemáticas con el mundo real: } \\
\text { cuentos, imágenes, sellos con inspiración } \\
\text { matemática, canciones, usos en el arte, la medicina o } \\
\text { el deporte. También se estructura por niveles } \\
\text { educativos. Perfecta para curiosear y extraer un } \\
\text { montón de materiales para la clase. }\end{array}$ \\
\hline & $\begin{array}{l}\text { Matemáticas de } \\
\text { cine }\end{array}$ & $\begin{array}{l}\text { Blog del profesor Ángel Requena Fraile, dedicado a } \\
\text { comentar y recomendar películas en las que los } \\
\text { conceptos matemáticos tienen mayor o menor } \\
\text { protagonismo. Una forma de afrontar la asignatura a } \\
\text { través del séptimo arte. }\end{array}$ \\
\hline & $\begin{array}{l}\text { Experiencing } \\
\text { Maths. }\end{array}$ & $\begin{array}{l}\text { Minisite con propuestas educativas para poner en } \\
\text { práctica las Matemáticas observando el mundo que } \\
\text { nos rodea e interactuando con él. }\end{array}$ \\
\hline & $\begin{array}{l}\text { Pasatiempos y } \\
\text { juegos en clase } \\
\text { de matemáticas. }\end{array}$ & $\begin{array}{l}\text { Recopilación de recursos lúdicos matemáticos para } \\
\text { tercer ciclo de Primaria, Secundaria y Bachillerato que } \\
\text { utilizan las matemáticas en situaciones de la vida } \\
\text { cotidiana. Otra buena opción es Pasatiempos } \\
\text { matemáticos de la prensa, donde se recopilan } \\
\text { pasatiempos aparecidos en periódicos o revistas cuya } \\
\text { resolución se realiza a partir de conceptos u } \\
\text { operaciones matemáticas }\end{array}$ \\
\hline
\end{tabular}

\section{Métodos}

La investigación tuvo un enfoque cuanti-cualitativo, es decir, que se abordó el tema de estudio desde los aspectos cuantitativos y medibles, con la finalidad de identificar hallazgos de importancia que sirvan de base a la investigación en lo relativo a la aplicación de las plataformas tecnológicas en el área de matemática. De la misma manera, se aplicó la investigación bibliográfica, porque se procedió al análisis de textos especializados, respecto al objeto en estudio, considerando aquellos producidos durante la última década para tener información mucho más actualizada.

Los métodos utilizados para la investigación son: analítico y deductivo partiendo de los conceptos generales se identificaron los 
componentes que a su vez son estudiado por separado, así se logra determinar la herramienta necesaria para el análisis de las plataformas tecnológicas. Métodos que sirvieron para familiarizarse con fenómenos conocidos en el campo de la educación en primero de bachillerato, para que se pueda obtener información que permita la posibilidad de llevar a cabo una investigación de las plataformas tecnológicas en los contextos virtuales.

La población objeto de estudio correspondió a expertos en plataformas tecnológicas, docentes y estudiantes de la Unidad Educativa San Mateo del primer año de bachillerato, ubicada en la zona rural de la ciudad de Manta, Unidad Educativa San Mateo de primero de bachillerato.

El muestreo por conveniencia, fue utilizado en la investigación cuantitativa, permitiendo escoger criterios para la determinación de la muestra. Mismo que permitió la selección de los docentes que imparten clase de matemáticas, también a los estudiantes de primero de bachillerato y expertos en plataformas tecnológicas. Con esta explicación se consideró a 12 docentes de matemáticas, al igual que la totalidad del centro educativo seleccionado; 75 estudiantes de primero bachillerato y 1 expertos en plataformas tecnológicas,

La información primaria fue proporcionada por los docentes de la Unidad Educativa San Mateo de primero de bachillerato, como fuente secundaria se contó con repositorios de Google académico de universidades, libros digitales, artículos científicos.

Las técnicas de procesamiento y análisis de los resultados, que se utilizaron fueron métodos estadísticos, mediante la recolección de datos por medio de cuestionarios de encuestas realizadas en Google Forms, con preguntas de base estructurada a docentes, estudiantes y la entrevista a expertos en plataformas tecnológicas, que permiten evidenciar los resultados de la investigación. 


\section{Resultados y discusión}

Tabla 1. Importancia de las plataformas tecnológicas en la educación.

\begin{tabular}{cc}
\hline Alternativas & Porcentajes \\
\hline Importante & $83.3 \%$ \\
Poco importante & - \\
Medianamente importante & $16,7 \%$ \\
Nada importante & - \\
Me es indiferente & - \\
\hline Total & $\mathbf{1 0 0 \%}$
\end{tabular}

Nota. Fuente: Autor (2021), Unidad Educativa San Mateo.

Como se observa en la Tabla 1, los resultados determinan con un $83 \%$ que el uso de las plataformas tecnológicas es importante en el proceso de aprendizaje y enseñanza, se puede inferir que los docentes encuestados asumen la necesidad de involucrar las plataformas tecnológicas en el proceso educativo como parte de formación de los estudiantes para que le brinden herramientas que le permitan interactuar y tener una mejor comprensión en lo referente a las clases virtuales. La educación siempre brinda respuestas a las necesidades de la sociedad, donde el profesorado a través de las múltiples herramientas coadyuva en este proceso, para eso debe tener un amplio conocimiento sobre las materias que imparte, en el caso de las matemáticas apoyada en las plataformas digitales hace que se requieran docentes conocedores del uso técnico de las tecnologías con excelente empleo pedagógico de las mismas.

Barrera \& Guapi (2018):

Las plataformas virtuales se han convertido en una herramienta potente en la tecnología educativa, siendo capaz de desarrollar la independencia de los saberes en muchos casos y el acercamiento virtual entre docentes y dicentes. La tecnología educativa como modelo pedagógico se centra en la corriente psicológica del conductismo y que considera el 


aprendizaje básicamente en $\quad \begin{aligned} & \text { como resultado la enseñanza } \\ & \text { estímulos y respuestas, dando }\end{aligned}$ programada. (Pág. 4)

Tabla 2. Plataformas utilizadas para impartir las clases de matemáticas

\begin{tabular}{ccc}
\hline Alternativas & Docentes & $\%$ \\
\hline YouTube & 0 & 0 \\
Zoom & 5 & 41.7 \\
Moodle & 0 & 0 \\
Microsoft Teams & 6 & 50.0 \\
Blackboard & 0 & 0 \\
WhatsApp Web & 1 & 8.3 \\
Ninguno & 0 & 0 \\
\hline Total & $\mathbf{1 2}$ & $\mathbf{1 0 0 \%}$ \\
\hline
\end{tabular}

Nota. Fuente: Autor (2021), Unidad Educativa San Mateo.

Logramos observar en la tabla 2 que los resultados indican que el $50 \%$ de los docentes imparten clases de matemáticas por Microsoft Teams, y el $41,7 \%$ utilizan Zoom, considerando que las dos opciones mayoritariamente escogidas son plataformas que fueron creadas originalmente con características empresariales y no con fines educativos.

Sin embargo, ante la demanda de la educación en línea, la plataforma tecnológica elegida para el proceso de enseñanza por el docente debe estar al alcance de los estudiantes para impartir las clases de matemáticas y así lograr que llegue de forma efectiva en el proceso de aprendizaje, de tal manera que se sientan en un entorno de confianza y de ser posible apoyado en juegos o actividades interactivas, características de la plataforma que apoyen a la construcción del conocimiento, permitiéndole al docente ser guía y apoyo para el uso idóneo y positivo en los educandos, padres de familia, directivos y la sociedad.

Sanchez (2020):

La plataforma de Zoom, que es una plataforma, un software, para video conferencias, creada varios años atrás, pero muy útil 
actualmente en pandemia ha adquirido una importancia debido a su facilidad de uso, al ser compatible con todos los sistemas operativos y además se lo pueden utilizar en todo tipo de dispositivos digitales. (Pág. 2)

Tabla 3. Conocimiento sobre las plataformas tecnológicas de parte de los docentes

\begin{tabular}{ccc}
\hline No. & Alternativas & Porcentajes \\
\hline 1 & Conocen y aplican & $8.3 \%$ \\
2 & Conocen, pero no aplican & $25.0 \%$ \\
3 & Desconocen y desean aprender & $66,7 \%$ \\
4 & No conocen y no desean aprender & $0 \%$ \\
5 & Es indiferente & $0 \%$ \\
\hline & Total & $\mathbf{1 0 0} \%$ \\
\hline
\end{tabular}

Nota. Fuente: Autor (2021), Unidad Educativa San Mateo.

Según el resultado obtenido en la tabla 3 sobre el conocimiento de las plataformas tecnológicas el 66,7\% de los docentes desconocen y desean aprender lo cual indica que el docente no tiene conocimiento, pero a su vez aspira aprender el manejo y proceso que se desarrolla en las plataformas tecnológicas.

La tecnología a medida que pasa el tiempo va evolucionando ganando espacio en los diversos entornos de la vida laboral, social, y lógicamente la educación no escapa a esta realidad, aunque a muchos docentes aún les cuesta entrar en esa dinámica virtual; una realidad que no puede ser desperdiciada al derivar en un amplio universo de conocimientos tanto para docentes como para toda la comunidad educativa, lo cual hace que los educadores deban tener una mejor preparación para hacer frente a este nuevo reto que se presenta, recodando que el proceso educativo es flexible por naturaleza, situación que los motiva a estar actualizados en sus conocimientos.

Padilla \& Conde-Carmona (2019):

La tecnología contribuye en la formación de profesores competentes, a nivel social $y$ personal, $y$ acorde a las exigencias del mundo actual; no obstante, siendo esta la era digital, es claro que el tipo de estudiantes de hoy no tienen similitudes con aquellos de 
décadas pasadas; es por ello que sus principales expectativas están alineadas a estímulos $y$ motivaciones que los profesores generen. (Pág. 117)

Tabla 4. Diseño de una plataforma tecnológica para la asignatura de matemáticas

\begin{tabular}{ccc}
\hline No. & Alternativas & Porcentajes \\
\hline 1 & $\mathrm{Si}$ & $100.0 \%$ \\
2 & No & $0.0 \%$ \\
\hline & Total & $\mathbf{1 0 0} \%$ \\
\hline
\end{tabular}

Nota. Fuente: Autor (2021), Unidad Educativa San Mateo.

En la tabla 4 se logra observar que el $100 \%$ de los docentes considera que se debe diseñar una plataforma tecnológica con característica que contenga contenidos y actividades de la asignatura de matemática. Esto es positivo desde todo punto de vista porque el aprendizaje de la esta materia permite aprovechar las nuevas tecnologías, se presentarían conceptos de manera mucho más visual e interactiva, además permite relacionarla con otros aspectos de la vida, de esa manera resultan accesibles para los estudiantes. Así también añaden un componente lúdico que las vuelve más atractivas.
Jiménez (2019):

En la compresión del lenguaje matemático, no basta con saber el algoritmo de memoria, se necesita que el estudiante contextualice la información y la aplique efectivamente en una situación problema, lo que evidentemente, no se puede lograr con tan solo la información, es necesario, que, mediante el uso adecuado de las TIC, el concepto matemático abstracto se formalice $y$ materialice. (Pág. 8)

Tabla 5. Plataformas tecnológicas utilizadas para impartir clases de matemáticas

\begin{tabular}{ccc}
\hline No. & Alternativas & Porcentajes \\
\hline 1 & YouTube & $2.7 \%$ \\
2 & Zoom & $80.0 \%$ \\
3 & Moodle & $0 \%$ \\
4 & Microsoft Teams & $16.0 \%$ \\
5 & Blackboard & $1.3 \%$
\end{tabular}




\begin{tabular}{ccc}
\hline No. & Alternativas & Porcentajes \\
\hline 6 & WhatsApp Web & $0 \%$ \\
7 & Ninguno & $0 \%$ \\
\hline & Total & $\mathbf{1 0 0 \%}$ \\
\hline
\end{tabular}

Nota. Fuente: Autor (2021), Unidad Educativa San Mateo.

En la tabla 5 se da a conocer que el $80 \%$ de los docentes utiliza la herramienta zoom para impartir la clase de matemática. Es imperativo contar con herramientas tecnológicas para la clase de matemáticas, por cuanto facilita la interacción entre estudiantes, permite a los docentes retroalimentar constantemente, y dejar sugerencias de textos o videos a través de enlaces digital.

Mendoza, Burbano\& Valdivieso (2019):
Los entornos interactivos de aprendizaje mediados por las TIC con el apoyo eficiente del tutor, generan beneficios cognitivos en los estudiantes para adquirir competencias matemáticas, siempre que se apliquen modelos didácticos pertinentes, asociados con metodologías activas $y$ constructivas que promuevan el aprendizaje significativo. (Pág.

Tabla 6. Herramientas utilizadas para impartir la clase de matemática

\begin{tabular}{lc}
\hline Alternativas & $\%$ \\
\hline Videos Interactivos & 72,6 \\
Presentaciones interactivas & 67,1 \\
Glosarios & 5,5 \\
Wikis & - \\
Actividades de arrastrar y soltar & 11 \\
Juegos matemáticos & 47,9 \\
Ninguna de las anteriores & 2,7 \\
\hline Total & $\mathbf{1 0 0} \%$ \\
\hline
\end{tabular}

Nota. Fuente: Autor (2021), Unidad Educativa San Mateo. 
En la tabla 6 se logra observar que los recursos más utilizados por los docentes para la impartición de clases de matemáticas nos indica que el 72.6 \% utilizó como recursos videos interactivos $67.1 \%$ presentaciones interactivas $47.9 \%$ juegos matemáticos $11 \%$ actividades de arrastrar y soltar $5.5 \%$ glosarios. Los diversos recursos disponibles para la clase de matemáticas existentes en la web, tienen que complementarse con la creatividad del docente que de una manera lúdica educar a los estudiantes, sumando al hecho de enseñar a que ellos sean creativos.

Cevallos, Lucas, Paredes \& Tomalá (2019):

El proceso de aprendizaje debe ser agradable para lograr un aprendizaje significativo, además, la motivación mediante el uso de la tecnología en los estudiantes cuando están aprendiendo es esencial para captar su atención y al mismo tiempo, los maestros innovan su forma de dar clases. (Pág. 86)

Tabla 7. Características debe tener una plataforma tecnológica en el área de matemáticas

\begin{tabular}{lll}
\hline \multicolumn{1}{c}{ No. } & \multicolumn{1}{c}{ Alternativas } & Porcentajes \\
\hline 1 & Plataforma de uso libre & $51.4 \%$ \\
2 & Que se pueda Incrustar recursos y actividades de otras & $41.9 \%$ \\
3 & plataformas & \\
& Accesible desde laptop, computadoras de escritorios y & $47.3 \%$ \\
4 & dispositivos móviles con capacidades mínima de hardware & \\
5 & Que permitan el monitoreo de avance de los estudiantes & $13.5 \%$ \\
7 & Que los estudiantes puedan recibir retroalimentación & $13.5 \%$ \\
8 & Capacidad de importar actividades y recursos & $32.4 \%$ \\
9 & Que los estudiantes puedan ver sus calificaciones & $25.7 \%$ \\
& Que se pueda agregar recursos & $18.9 \%$ \\
& Que disponga de herramientas para la interacción como & $45.9 \%$ \\
\hline
\end{tabular}

Nota. Fuente: Autor (2021), Unidad Educativa San Mateo.

En la tabla 7 se demuestran los datos obtenidos sobre la característica le gustaría que tuviera la plataforma tecnológica de matemática $51.4 \%$ que sea una plataforma libre $47.3 \%$ que sea accesible desde laptop computadora 
de escritorio y dispositivos móviles, $45.9 \%$ que contengan videos interactivos y $31 \%$ que se pueda enviar las actividades a las plataformas y32.4 que puedan ver sus calificaciones $25.7 \%$ que puedas dejar tu opinión y preguntas $18.9 \%$ que disponga de interacción como chat y foros $13.5 \%$ que se pueda recibir retroalimentación y permita el monitoreo de tus actividades.

La plataforma tiene que ser totalmente interactiva, de tal forma que los estudiantes puedan manejarla sin ninguna dificultad, incluso si ellos estiman conveniente sugerir mejoras sean incorporadas, tomando en consideración que el principio rector que debe primar para el funcionamiento de las plataformas es la comprensión de los educandos.

Según Medina (2013) con respecto a las plataformas virtuales y sus aplicaciones:

El uso de plataformas virtuales en los procesos educativos, especialmente a nivel de la educación superior, cada día toma más protagonismo en las propuestas metodológicas aplicadas. Las plataformas virtuales ofrecen aplicaciones con la utilización de las técnicas de la información y la comunicación, brindan las opciones integradoras de múltiples recursos dando paso al proceso de la digitalización. (Pág. 24)

Tabla 8. Implementar una plataforma tecnológica para matemáticas

\begin{tabular}{ccc}
\hline No. & Alternativas & Porcentajes \\
\hline 1 & $\mathrm{Si}$ & $97.3 \%$ \\
2 & $\mathrm{No}$ & $2.7 \%$ \\
\hline & Total & $\mathbf{1 0 0} \%$ \\
\hline
\end{tabular}

Nota. Fuente: Autor (2021), Unidad Educativa San Mateo.

En base a la tabla 8, los estudiantes están dispuestos a utilizar una plataforma tecnológica para el área de matemática el $97.3 \%$. Los estudiantes gustan de hacer uso de las computadoras, en el caso de la plataforma se deberían incorporar interactividad por medio de links que los lleve a portales electrónicos donde estén videos, y todo tipo de herramientas digitales que los motiven al aprendizaje. 
Covarrubias (2021):

La injerencia de las TICs en los procesos educativos vino a dejar especial relevancia, pues en cada rincón del mundo se pretendía la mejora de la calidad del proceso enseñanza-aprendizaje, de tal forma que su aplicación en el campo pedagógico buscaba optimizar los resultados de todo un sistema escolar y al mismo tiempo, llevar el conocimiento a las poblaciones particularmente excluidas. (Pág.152)

\section{Discusión}

Según Melchor la plataforma Zoom ha adquirido importancia en esta pandemia por su uso, en la presente investigación se obtuvo como resultado que con un $50 \%$ Microsoft Teams es la plataforma más utilizada para impartir clases en la asignatura de matemática. (Tabla 2 encuesta a docentes) Mendoza indica, los entornos interactivos de aprendizaje mediados por las TIC con el apoyo eficiente del tutor, generan beneficios cognitivos esto se apoya en el resultado de la encuesta a los estudiantes, ellos afirman, recibir clase en un $80 \%$ en la plataforma Zoom.
Podemos observar que las plataformas más utilizadas para impartir clases son Teams y Zoom indicándonos que los entornos virtuales están presentes en la actualidad en los procesos de enseñanzas en diferentes plataformas con el objetivo de elevar la calidad del proceso educativo, desarrollando campos virtuales y enseñanza en línea.

Por otro lado Jiménez expresa que en la comprensión del lenguaje matemático, no basta con saber algoritmo de memoria, se necesita que El estudiante contextualice la información y la aplique en una situación de problema, en la presente investigación se obtuvo como resultado que El 100\% de los docentes consideran que se debe diseñar plataformas tecnológicas con característica que contenga contenido y actividades de la asignatura de matemática que permita el desarrollo de habilidades en la resolución de problemas.

Por consiguiente Salazar expone que el aprendizaje debe ser agradable para lograr un aprendizaje significativo, en la presente investigación se obtuvo que el $72.6 \%$ de los docentes utilizan 
videos interactivos para el desarrollo de las clases, Medina expresa el uso de plataformas virtuales en los procesos educativos, especialmente a nivel de la educación superior deben tener aplicaciones con utilización de TIC y brindar opciones integradoras de múltiples recursos integrando los siguiente resultados obtenidos en la investigación el 47.3\% Que las plataformas tecnológicas deben brindar una fácil accesibilidad desde laptop, computadoras de escritorios y dispositivos móviles con capacidades mínima de hardware. El $51.4 \%$ considera que la principal característica de las plataformas tecnológicas deberían ser plataformas de uso libre. El $41.9 \%$ considera que en la plataforma se puedan enviar las actividades. (Tabla 6 encuesta a estudiantes).

Se debe observar que Padilla \& Conde-Cardona señalan que la tecnología contribuye en la formación de profesores competentes, a nivel social $y$ personal, y acorde a las exigencias del mundo actual apoyándose en la investigación los resultado exponen que el $66.7 \%$ de los docentes desconoce y desea aprender sobre las plataformas tecnológicas en el área de matemática; Hernández comenta que las TIC en los procesos educativos tienen especial relevancia, pues se pretende mejorar de la calidad del proceso enseñanza-aprendizaje, de tal forma que su aplicación en el campo pedagógico busca optimizar los resultados de todo un sistema escolar se puede observar en el resultado de la investigación que el 97.3. \% de los estudiantes estaría dispuesto a utilizarla una plataforma tecnológica con herramientas de matemática, resaltando la importancia de las plataformas tecnológicas en el proceso de enseñanza y de aprendizaje con el objetivo de impartir y desarrollar conocimiento en el área de matemáticas.

\section{Conclusiones}

Resulta necesario aprovechar las plataformas tecnológicas para desarrollar contenido que mejoren el proceso de enseñanza y de aprendizaje en el área de matemática, no es suficiente utilizar herramientas de video conferencias. Por tanto, es urgente desarrollar aulas virtuales personalizadas con 
recursos digitales interactivos aplicables en el área de las matemáticas, todo esto alineado a los contenidos y objetivos de la materia.

Debido a que el objeto de estudio fue en una institución fiscal, donde los estudiantes provienen de hogares con escasos recursos económicos, estos manifestaron que las plataformas virtuales deben ser accesibles desde dispositivos con características básicas de hardware, por lo que las plataformas virtuales deben adicionalmente ser compatibles con estos tipos de dispositivos.

Resulta importante resaltar la disposición de los estudiantes para utilizar plataformas tecnológicas desarrolladas especialmente para el área de matemática. Cabe mencionar la responsabilidad que tienen las autoridades de la institución de educación, en idear mecanismos que favorezcan al desarrollo de estas plataformas y a la capacitación de los docentes y estudiantes, solo así lograremos adaptarnos a los cambios innegables que dejado el uso de la tecnología en el ámbito educativo.

\section{Bibliografía}

Aida Cañar; María F. Andrango \& Edwin Muso. (2021). La educación del futuro mediante plataformas virtuales. Dominio de la Ciencia, 1223.

Anayda Fernández \& Miguel Rivero. (2014). Las plataformas de aprendizajes, una alternativa a tener en cuenta en el proceso de enseñanza aprendizaje. Revista cubana de información médica, 208.

Aulaplaneta. (2015). Aulaplaneta. Obtenido de 25 herramientas para enseñar Matemáticas con las TIC [Infografía].: https://bit.ly/3wGKzAm

Avalos E., V. S. (2020). La plataforma Schoology en el aprendizaje de la matemática en estudiantes secundarios. INNOVA Research Journal, 135.

Barrera, V. G. (2018). La importancia del uso de las plataformas virtuales en la educación superior. Revista Atlante: Cuadernos de Educación y Desarrollo. Obtenido de https://bit.ly/2RZmhCM

Carignano, F. (2012). Magnífica Tecnología. Revista Científica de la asociación medica de bahía blanca, 22(1). Obtenido de

https://www.ambb.org.ar/ojs/i ndex.php/RCAMBB/article/vie w/59 
Cevallos, J., Lucas, X., Paredes, J., \& Tomalá, J. (2019). Uso de herramientas tecnológicas en el aula para generar motivación en estudiantes del noveno de básica de las unidades educativas Walt Whitman, Salinas y Simón Bolívar, Ecuador. CPI Ciencias Pedagógicas e Innovación, 86-93.

Cobos, J., \& Torres, P. (2017). Tecnología educativa y su papel en el logro de los fines de la educación. Educere, 38.

Covarrubias, L. (2021). Educación a distancia: transformación de los aprendizajes. Telos, 150160.

Ivan Padilla \& Rovion CondeCarmona. (2019). Uso y formación en TIC en profesores de matemáticas: un análisis cualitativo. Revista Virtual Católica del Norte, 116-136. Obtenido de https://bit.ly/3xQtwxj

Jiménez, D. (2019). Herramientas digitales para la enseñanza de las matemáticas en la educación básica. Bogotá: Universidad Cooperativa de Colombia. Obtenido de https://bit.ly/3baDuzR

Medina, W. (2013). Evaluación del uso de las plataformas virtuales. Guayaquil: Universidad de Guayaquil.
Melchor, Sánchez Mediola. (2020). Involucrar a los estudiantes al usar Zoom. Universidad Nacional Autónoma de México, 2.

Mendoza, B., \& Valdivieso, M. (2019). El papel del docente de matemáticas en Educación superior a distancia y virtual: una mirada desde los métodos mixtos de investigación. Revista Espacios. Obtenido de https://bit.ly/2QSTUpS

Mendoza, H., Burbano, V., \& Valdivieso, M. (2019.). El Rol del Docente de Matemáticas en Educación Virtual Universitaria. Un Estudio en la Universidad Pedagógica y Tecnológica de Colombia. Form. Univ. vol.12 no.5., 5160. Obtenido de https://bit.ly/3tkU46s

Raúl Campos; Gloria Boulet. (2016). Las Plataformas tecnológicas en universidad contemporánea. Atenas: Redalyc.

Sánchez, J. (2005). Plataformas tecnológicas para el entorno educativo.

Acción Pedagógica, 18-24.

Universidad Internacional de Valencia. (2015). Obtenido de Características, tipos y plataformas más utilizadas para estudiar a distancia.: https://bit.ly/2SAaJGx 